\title{
DETERMINATION OF DYNAMIC CHARACTERISTICS OF HEAT FIRE DETECTORS
}

\author{
Yuriy Abramov \\ Scientific and Research Center \\ National University of Civil Defence of Ukraine \\ 94 Chernyshevskaya str., Kharkiv, Ukraine, 61023 \\ zxcv600@ukr.net \\ Yaroslav Kalchenko \\ Scientific Department on Problems of Civil Protection and Technologic and Ecological Safety of \\ the Scientific and Research Center \\ National University of Civil Defence of Ukraine \\ 94 Chernyshevskaya str., Kharkiv, Ukraine, 61023 \\ axe2114@gmail.com \\ Olena Liashevska \\ Department of Management and Civil Protection Organization \\ National University of Civil Defence of Ukraine \\ 94 Chernyshevskaya str., Kharkiv, Ukraine, 61023 \\ elenalyashevskaya959@gmail.com
}

\begin{abstract}
The proposed methods for determining the dynamic characteristics of heat fire detectors in the time and frequency domains, focused on the use of existing thermal chambers. The proposed method for determining the transition function of the detector is implemented as follows. Heat fire detector creates a thermal effect in the form of a linearly increasing function. The response of the output signal to the influence of this type is measured and approximated using the Heaviside function at regular intervals.

It is shown that information on the transition function of a heat fire detector can be used to determine its frequency characteristics by approximating it with Heaviside functions at the same time intervals. This method of determining the frequency characteristics will significantly reduce the time to determine them compared to the classical method, and also eliminate the need for additional equipment.

As a result of the studies, the choice of the sampling interval was justified on the example of a class A1 heatfire detector and certain sampling intervals for determining their transition function $\left(\tau_{0} \leq 1.05 \mathrm{~s}\right)$, amplitude-frequency characteristic $\left(\tau_{0} \leq 0.27 \mathrm{~s}\right)$ and phase-frequency characteristic $\left(\tau_{0} \leq 2.0 \mathrm{~s}\right)$.

The proposed methods for determining the dynamic characteristics of heat fire detectors open up new opportunities for developing methods for monitoring their technical condition. This is because the information about the transition function of the detector can be used in two ways. The first method involves comparing a certain transition function of the detector with an exemplary one. The second method consists in determining other characteristics of the detector based on information about its transient function and comparing them with standard values.
\end{abstract}

Keywords: heat fire detector, transient function, frequency characteristics, sampling interval.

\section{Introduction}

One of the problems in the way of increasing the efficiency of fire detection at an early stage of a fire is the improvement of its operation system, in particular, methods for monitoring the technical condition of heat fire detectors (HFD). In a series of European standards EN 54-5, the American standard NFPA 72 and the Russian GOST 53325-2009, methods are described for monitoring the technical state of the HFD [1], which is carried out by conducting tests in a heat channel or test hot spots. The purpose of such tests is determination of the time and temperature of the detector. Methods for determining the HFD response time are considered in [2-4], and the calculation method in accordance with NFPA 72 is given in [5], where it is shown that the detector response time depends on the value of its constant time. In Korea, the time constant of heat fire detectors 
is determined during their testing in heat chambers and is used to predict their performance [6]. In $[7,8]$ it is shown how the HFD time constant can be used to determine its response time. However, it should be noted that not all possibilities have been used in monitoring the HFD technical state. In particular, it is considered an open question about the use of the HFD dynamic characteristics to assess their technical condition. At the same time, it is necessary to keep in mind that the classical methods for identifying the HFD dynamic characteristics, both in the time and in the frequency domain, have specific features. A number of such features preclude the use of the dynamic HFD characteristics to assess their technical condition. For example, the definition of the HFD transition function necessitates the formation of a test impact described by the Heaviside function. With regard to high-speed HFD, such an approach is associated with the presence of a sufficiently large error due to the inertia of thermal processes. The proliferation of classical methods for determining the HFD frequency characteristics involves complex equipment, as well as time-consuming. A common disadvantage of methods for determining the HFD dynamic characteristics is that their implementation requires the use of additional and very specific equipment.

In this regard, the aim of research is development of methods for determining the dynamic characteristics of heat fire detectors in the time and frequency domains, focused on the use of existing thermal chambers.

To achieve this aim it is necessary to solve the following objectives:

- develop a method for determining the transition function of a heat fire detector by approximating its output signal when it is influenced by a linearly increasing heat flux;

- develop a method for determining the frequency characteristics of a heat fire detector, the initial information for which is the approximation of its transition function;

- justify the choice of the sampling interval when determining the transition function and frequency characteristics of heat fire detectors.

\section{Determination of the transition function of heat fire detectors}

The HFD installed in the working volume of the heat chamber is exposed to a test signal in the form of a temperature increment $\Delta \mathrm{T}(\mathrm{t})$ relative to the initial value $\mathrm{T}_{0}$, which is linearly modified

$$
\Delta \mathrm{T}(\mathrm{t})=\mathrm{a} \cdot \mathrm{t}
$$

where $\mathrm{a}$ - the temperature growth rate in the working area of the heat chamber; $\mathrm{t}$ - time.

With such an effect, the HFD output signal TPI $\theta_{1}(\mathrm{t})$ will change, as shown in Fig. 1 [9].

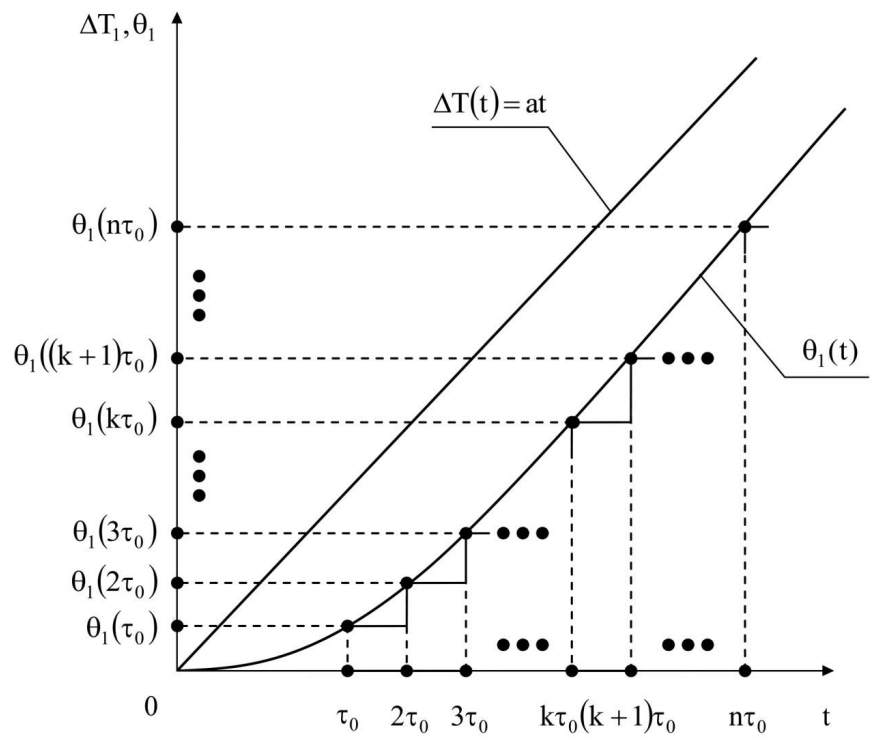

Fig. 1. The dependence of the temperature increment $T(t)$ on the time in the heat chamber $T(t)$ and the sensitive element of the heat detector $\theta_{1}(t)$ 
The expression for this signal is

$$
\theta_{1}(\mathrm{t})=\mathrm{L}^{-1}[\mathrm{~W}(\mathrm{p}) \Delta \mathrm{T}(\mathrm{p})]=\mathrm{L}^{-1}\left[\mathrm{~W}(\mathrm{p}) \mathrm{ap}^{-2}\right],
$$

where $\mathrm{L}^{-1}$ - the operator of the inverse integral Laplace transform; W(p) - HFD transfer function; $\mathrm{p}$ - a complex number.

The HFD transfer function is described by the expression

$$
\mathrm{W}(\mathrm{p})=(\tau \mathrm{p}+1)^{-1},
$$

where $\tau$ - the time constant.

Taking into account (3) the HFD output signal $\theta_{1}(\mathrm{t})$, is transformed to the form

$$
\theta_{1}(\mathrm{t})=\mathrm{a}\left[\mathrm{t}-\tau\left(1-\exp \left(-\frac{\mathrm{t}}{\tau}\right)\right)\right]=\mathrm{a}\left[\mathrm{t}-\tau \theta_{0}(\mathrm{t})\right]
$$

where $\theta_{0}(\mathrm{t})-$ HFD unit transition function.

For $\theta_{0}(\mathrm{t})-$ the relation

$$
\theta_{0}(t)=L^{-1}\left[W(p) p^{-1}\right]=L^{-1}\left[[(\tau p+1) p]^{-1}\right]=1-\exp \left(-\frac{t}{\tau}\right)
$$

From (4) and (5) follows the dependence for the function $\theta_{0}(\mathrm{t})$

$$
\tau \theta_{0}(\mathrm{t})=\mathrm{H}(\mathrm{t})=\mathrm{t}-\mathrm{a}^{-1} \theta_{1}(\mathrm{t})
$$

where $H(t)$ - HFD transition function, which differs from the single transition function $\theta_{0}(t)$ by scale.

If at the moments of time that are lagging from each other by the same a priori given interval $\tau_{0}$, HFD output signal is measured against its value at the previous time, that is,

$$
\Delta \theta_{\mathrm{lk}}=\theta_{1}\left(\mathrm{k} \tau_{0}\right)-\theta_{1}\left((\mathrm{k}-1) \tau_{0}\right) ; \mathrm{k}=\overline{\mathrm{l}, \mathrm{n}},
$$

then for the output signal $\theta_{1}(t)$ will take place

$$
\theta_{1}(\mathrm{t})=\sum_{\mathrm{k}=1}^{\mathrm{n}} \Delta \theta_{\mathrm{lk}} \cdot 1\left(\mathrm{t}-\mathrm{k} \tau_{0}\right)
$$

where $1\left(\mathrm{t}-\mathrm{k} \tau_{0}\right)-$ Heaviside function.

Taking into account (8), the expression for the HFD transition function (6) is transformed as follows

$$
\mathrm{H}(\mathrm{t})=\mathrm{t}-\mathrm{a}^{-1} \sum_{\mathrm{k}=1}^{\mathrm{n}} \Delta \theta_{\mathrm{lk}} \cdot 1\left(\mathrm{t}-\mathrm{k} \tau_{0}\right)
$$

The number of measurements of the HFD output signal $\mathrm{n}$ is determined from the expression

$$
\mathrm{n} \tau_{\theta}=\mathrm{t}
$$

where $\mathrm{t}_{\mathrm{c}}$ - the detector response time, and $\tau_{0}$ - determined in accordance with the Kotelnikov theorem

$$
\tau_{0}=0,5 f_{\mathrm{m}}^{-1}
$$

where $f_{m}$ - the maximum frequency of the spectrum of the function $\theta_{1}(t)$. 
From expressions (10) and (11) follows the expression for the parameter $\mathrm{n}$

$$
\mathrm{n}=2 \mathrm{f}_{\mathrm{m}} \mathrm{t}_{\mathrm{c}} \text {. }
$$

In this expression, the value of the parameter $t_{c}$ is selected depending on the magnitude of the temperature growth rate in the working volume of the thermal chamber and is normalized by the current standard. For example, for countries in which the current regulatory document is EN 54-5, for class A1 detectors with $\mathrm{a}=0.5^{\circ} \mathrm{C} / \mathrm{s}$, the maximum response time is $100 \mathrm{~s}$.

Thus, the algorithm for determining the HFD transition function is reduced to the following procedures:

- installation of the detector in the working area of the heat chamber;

- creation of a linearly increasing thermal effect on the detector, the rate of change of which is selected in accordance with the current regulatory document;

- measurement at each of the points in time that are a priori specified interval, that the output value of the detector is greater than the previous point in time;

- determination of the transition function of the detector according to the formula (9).

This method of determining the HFD transition function is universal and can be applied to detectors that are built using various physical principles. The advantage of this method is that its implementation can be carried out in existing thermal chambers, which are used to monitor the HFD technical state, so additional equipment is not required for the formation of a test exposure. It is also worth noting that information on the HFD transition function can be used both to monitor the HFD technical state and to determine its frequency characteristics.

However, when determining the HFD transition function with this method, the question of choosing the sampling interval remains open. When using the Kotelnikov theorem to select the sampling interval, there is uncertainty, which is caused by the ambiguity of choosing the maximum value of the spectral density of the input action. One of the options for removing such uncertainty may be to determine the sampling interval using an accuracy criterion.

Fig. 2 shows the graphs of the transition function of HFD class A1 with a time constant $\tau=20 \mathrm{~s}$ (EN 54-5), which were determined by formula (9) for different sampling intervals.

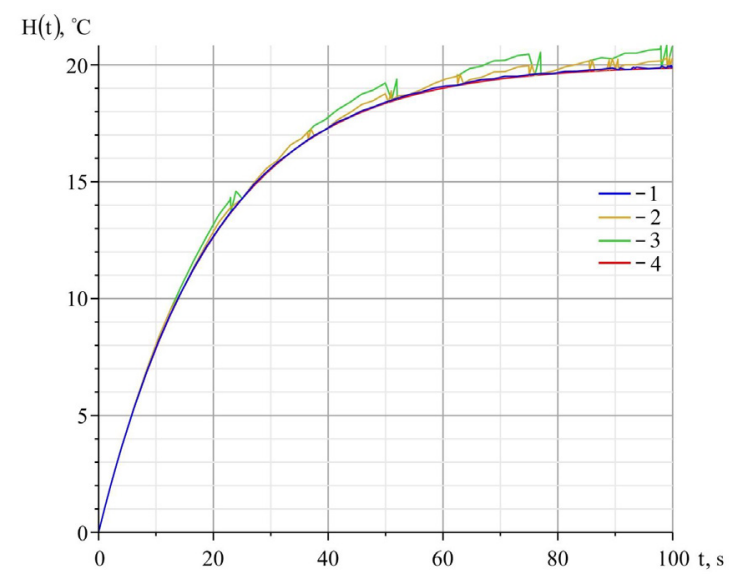

Fig. 2. Transitional functions of a heat fire detector

with a time constant $\tau=20 \mathrm{~s}$, determined at different sampling intervals:

$1-\tau_{0}=\mathrm{s} ; 2-\tau_{0}=0.5 \mathrm{~s} ; 3-\tau_{0}=1.0 \mathrm{~s} ; 4-$ exemplary transient function of the detector

The exemplary transient function of the detector is determined by the expression

$$
\theta(t)=K\left(1-\exp \left(-\frac{t}{\tau}\right)\right)
$$

where $\mathrm{K}$ - the coefficient (in the considered example, $\mathrm{K}=20^{\circ} \mathrm{C}$ ). 
The error in determining the transition function of the detector is determined by the expression

$$
\delta_{1}=\left|1-\frac{\mathrm{H}(\mathrm{t})}{\theta(\mathrm{t})}\right| .
$$

Fig. 3 shows the graphical dependence for the error $\delta_{1}$ when determining the HFD transition function with a time constant $\tau=20 \mathrm{~s}$ from the sampling interval.

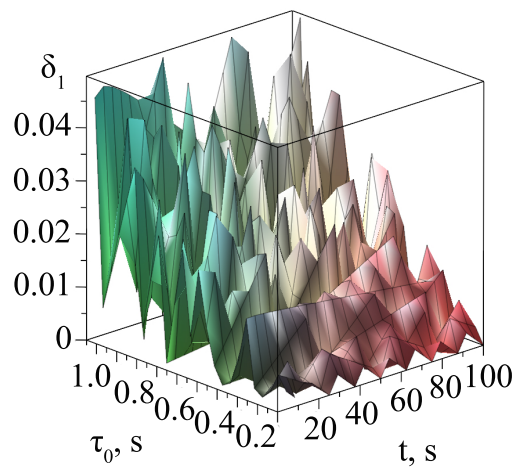

Fig. 3. The dependence of the magnitude of the error on the sampling interval for the transition function of a heat fire detector with a time constant $\tau=20 \mathrm{~s}$

Fig. 4 shows the dependence of the maximum value of the error $\delta_{1 \mathrm{M}}$ in determining the HFD transition function on the magnitude of the sampling interval and the approximation of this dependence, which is described by the expression

$$
\delta_{1 \mathrm{M}}=0,047 \cdot \tau_{0}
$$

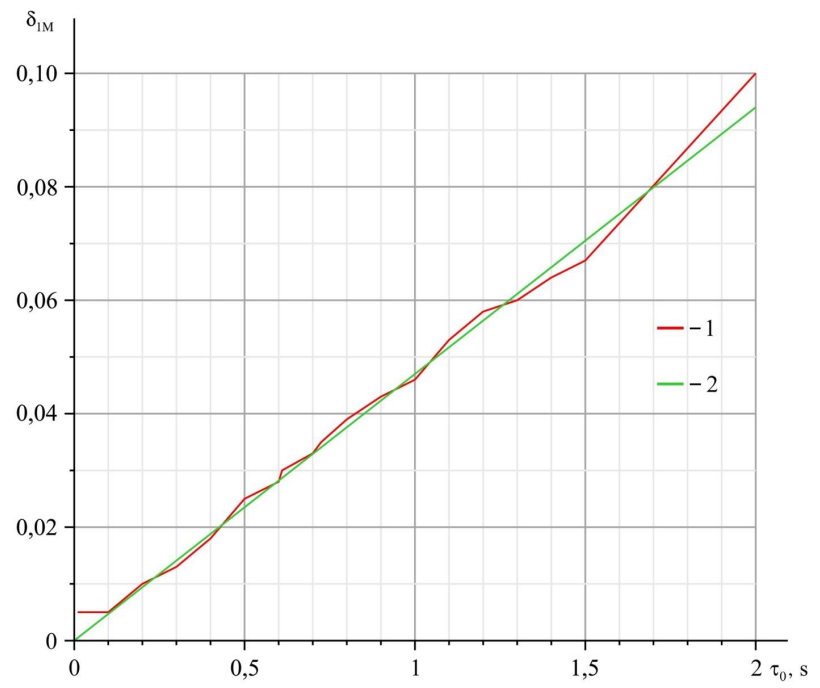

Fig. 4. The dependence of the magnitude of the error on the sampling interval for the transition function of a heat fire detector with a time constant $\tau=20 \mathrm{~s}$ : $1-$ a plot of the maximum error value in determining the transition function of the heat firedetector on the magnitude of the sampling interval; 2 - approximation of this dependence

From the analysis of dependencies presented in Fig. 4, it follows that for the HFD transition function with a time constant $\tau=20 \mathrm{~s}$ with an error not exceeding $5 \%$, it is necessary to use the sampling interval $\tau_{0} \leq 1,05 \mathrm{~s}$. 


\section{Determination of the frequency characteristics of heat fire detectors}

To determine the HFD frequency characteristics - the amplitude-frequency characteristic (AFC) and phase-frequency characteristic (PFC), the transition material is the starting material, which is determined according to (9). Such a transition function will correspond to the equivalent thermal effect in the form of a temperature difference $T_{1}-T_{0}$

$$
\Delta \mathrm{T}(\mathrm{t})=\left(\mathrm{T}_{1}-\mathrm{T}_{0}\right) \cdot 1(\mathrm{t}),
$$

where

$$
\mathrm{T}_{1}-\mathrm{T}_{0}=\lim _{\mathrm{t} \rightarrow \infty} \mathrm{H}(\mathrm{t}) .
$$

The function $\mathrm{H}(\mathrm{t})$ is approximated by the expression

$$
\mathrm{H}(\mathrm{t})=\sum_{\mathrm{k}=0}^{\mathrm{n}} \Delta \mathrm{H}_{\mathrm{k}} \cdot 1(\mathrm{t}-(\mathrm{k}+0,5) \Delta \mathrm{t})
$$

The graphical interpretation of such an approximation is explained in Fig. 5 [10].

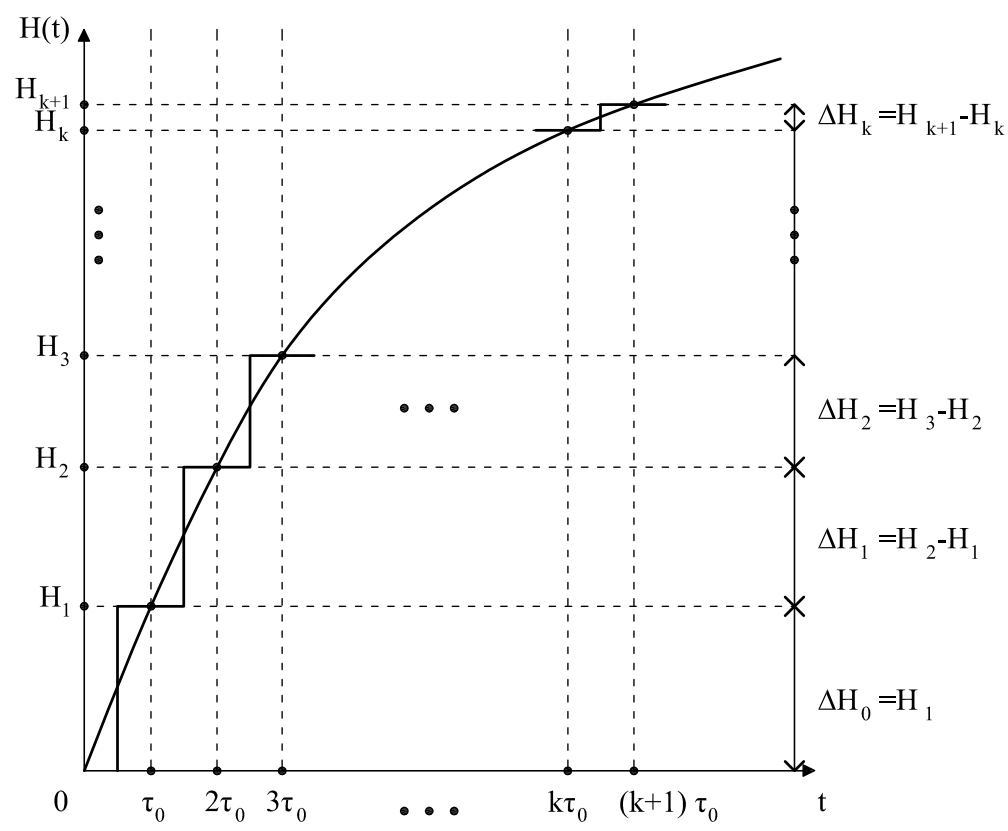

Fig. 5. The temperature of the sensing element of the heat fire detector relative to the initial value $\mathrm{T}_{0}$ when it is subjected to an abrupt change in temperature

Let's apply to the expression (18) the integral Laplace transform, after which

$$
\mathrm{H}(\mathrm{p})=\int_{0}^{\infty} \mathrm{H}(\mathrm{t}) \exp (-\mathrm{pt}) \mathrm{dt}=\mathrm{p}^{-1} \sum_{\mathrm{k}=0}^{\mathrm{n}} \Delta \mathrm{H}_{\mathrm{k}} \cdot \exp \left[-\mathrm{p}(\mathrm{k}+0,5) \tau_{0}\right]
$$

From (19) follows the expression for the transfer function of the heat fire detector

$$
\mathrm{W}(\mathrm{p})=\left(\mathrm{T}_{1}-\mathrm{T}_{0}\right)^{-1} \sum_{\mathrm{k}=0}^{\mathrm{n}} \Delta \mathrm{H}_{\mathrm{k}} \cdot \exp \left[-\mathrm{p}(\mathrm{k}+0,5) \tau_{0}\right] .
$$

From the expression (20) let's obtain the expression for the amplitude-phase frequency response of the detector 


$$
\begin{gathered}
\mathrm{W}(\mathrm{j} \omega)=\left(\mathrm{T}_{1}-\mathrm{T}_{0}\right)^{-1} \sum_{\mathrm{k}=0}^{\mathrm{n}} \Delta \mathrm{H}_{\mathrm{k}} \cdot \exp \left[-\mathrm{j} \omega(\mathrm{k}+0,5) \tau_{0}\right]= \\
=\left(\mathrm{T}_{1}-\mathrm{T}_{0}\right)^{-1} \sum_{\mathrm{k}=0}^{\mathrm{n}} \Delta \mathrm{H}_{\mathrm{k}}\left[\cos \left[\omega(\mathrm{k}+0,5) \tau_{0}\right]-\mathrm{j} \sin \left[\omega(\mathrm{k}+0,5) \tau_{0}\right]\right],
\end{gathered}
$$

where $\mathrm{j}$ - the imaginary unit; $\omega-$ the circular frequency.

Frequency response $\mathrm{A}(\omega))$ and phase response $\varphi(\omega)$ associated with the amplitude-phase frequency response $\mathrm{W}(\mathrm{j} \omega))$ as follows

$$
\begin{aligned}
& A(\omega)=\bmod W(j \omega) ; \\
& \phi(\omega)=\arg W(j \omega),
\end{aligned}
$$

which leads to expressions

$$
\begin{gathered}
A(\omega)=\left(T_{1}-T_{0}\right)^{-1}\left[\left(\sum_{k=0}^{n} \Delta H_{k} \cdot \cos \left[\omega(k+0,5) \tau_{0}\right]\right)^{2}+\right. \\
\left.+\left(\sum_{k=0}^{n} \Delta H_{k} \cdot \sin \left[\omega(k+0,5) \tau_{0}\right]\right)^{2}\right]^{0,5} ; \\
\varphi(\omega)=-\operatorname{arctg} \frac{\sum_{k=0}^{n} \Delta H_{k} \cdot \sin \left[\omega(k+0,5) \tau_{0}\right]}{\sum_{k=0}^{n} \Delta H_{k} \cdot \cos \left[\omega(k+0,5) \tau_{0}\right]}
\end{gathered}
$$

Thus, the algorithm for determining the HFD frequency characteristics is reduced to the following procedures:

- installation of the detector in the working area of the heat chamber;

- determination of the HFD transition function according to the algorithm given in section 2;

- approximation of the HFD transition function;

- determination of the HFD frequency characteristics by formulas (23) and (24).

To select the sampling interval in determining the HFD frequency characteristics, let's use a similar approach that is used in determining its transition function. To this end, let's determine the error

$$
\begin{aligned}
& \delta_{2 \mathrm{~A}}=\left|1-\frac{\mathrm{A}(\omega)}{\mathrm{A}_{0}(\omega)}\right| ; \\
& \delta_{2 \varphi}=\left|1-\frac{\varphi(\omega)}{\varphi_{0}(\omega)}\right|,
\end{aligned}
$$

where

$$
\begin{gathered}
A_{0}(\omega)=\left(1+\omega^{2} \tau^{2}\right)^{-0,5} ; \\
\varphi_{0}(\omega)=-\operatorname{arctg} \omega \tau .
\end{gathered}
$$

Fig. 6, 7 show the dependences for the errors $\delta_{2 \mathrm{~A}}$ and $\delta_{2 \varphi}$.. It should be noted that for $\tau_{0} \leq 2,00 \mathrm{~s}$ with the maximum error $\delta_{2 \varphi}$ does not exceed $3.2 \%$, and for the maximum error $\delta_{2 \mathrm{~A}}$ the dependence $\delta_{2 \mathrm{M}}=\delta_{2 \mathrm{M}}\left(\tau_{0}\right)$ has the form shown in Fig. 8. This dependence can be approximated by the expression 


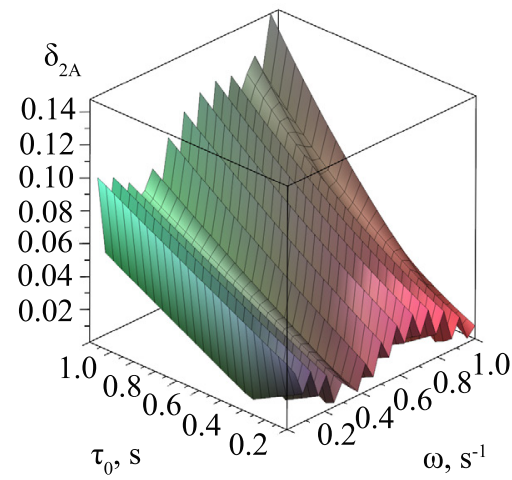

Fig. 6. Graph of the magnitude of the error in determining the amplitude-frequency characteristics of the heat fire detector on the magnitude of the sampling interval and circular frequency

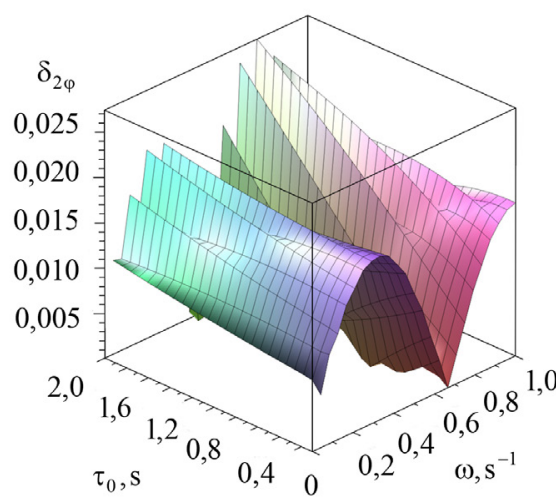

Fig. 7. Graph of the magnitude of the error in determining the phase-frequency characteristics of a heat fire detector on the magnitude of the sampling interval and circular frequency

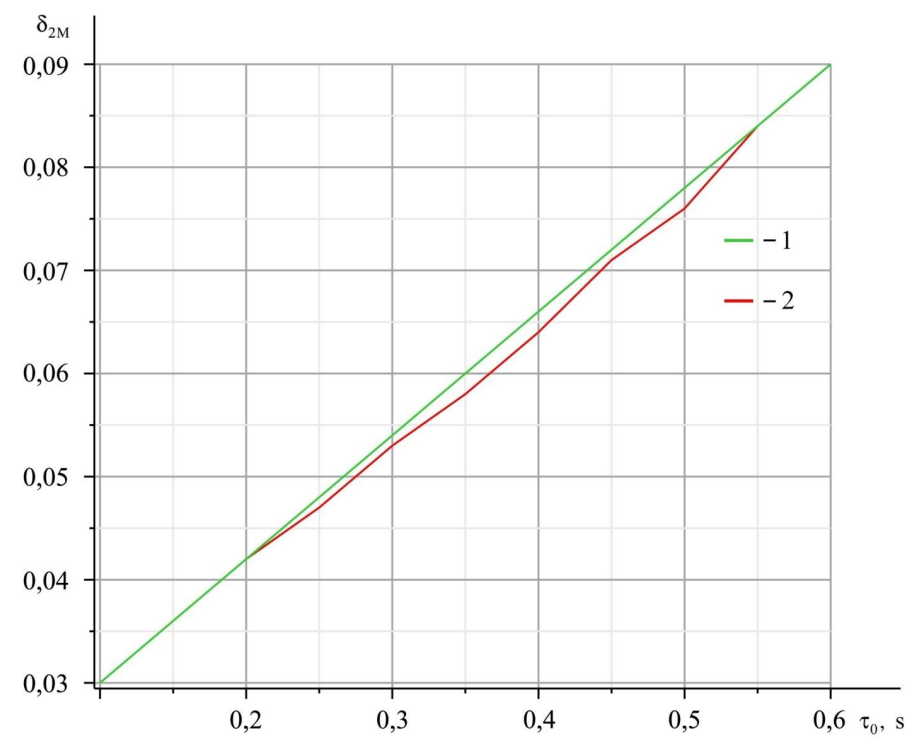

Fig. 8. The dependence of the error on the sampling interval when determining the amplitude-frequency characteristic of a heat fire detector with a time constant $\tau=20 \mathrm{~s}$ :

1 - a plot of the maximum error value in determining the amplitude-frequency characteristic of the heat fire detector on the sampling interval; 2 - approximation of this dependence 
From the analysis of the dependences shown in Fig. 8, it follows that to determine the HFD AFC in a time constant $\tau=20 \mathrm{~s}$, with an error not exceeding $5 \%$, it is necessary to use the sampling interval $\tau_{0} \leq 0,27 \mathrm{~s}$.

\section{Discussion of the research results to determine the dynamic characteristics of heat fire detectors}

To obtain the HFD dynamic characteristics in the time and frequency domains, an approach is used which is based on the formation of a thermal test effect as a linearly increasing function of time. Obtaining the HFD transition function implies approximation of its response to such a test-effect using the Heaviside function at equal intervals of time. These time intervals are selected in accordance with the permissible error value that meets the requirements of, in particular, EN 54-5. The data array, obtained by changing the HFD input signal, at discrete points in time, is used as input data in the algorithm for determining the HFD transition function. The advantage of this method is that the standard thermal chambers, provided by the NFPA 72 and EN 54-5 standards, are used to form a test exposure to the HFD.

To determine the HFD frequency characteristics, its transient function obtained using a test-action in the form of a linearly increasing function of time is used as the initial material. The algorithm for determining the HFD frequency characteristics provides for the approximation of such a transition function by Heaviside functions at equal intervals of time. These time intervals are determined taking into account the approximation error and in accordance with the requirements of EN 54-5. The advantage of this method of determining theHFD frequency characteristics is a reduction in its duration $n$ times compared to the classical method. This is because when implementing such a method, there is no need to measure the amplitude of the output signal at each frequency.

The following results are obtained:

- methodis developed for determining theHFD transition function, which is based on the approximation of the HFD response to the thermal test effect as a linearly increasing function of time;

- methodis developed for determining the HFD frequency characteristics, which involves the use of its transient function as transient data; acteristics;

- choice of the sampling interval is justified when determining the HFD dynamic char-

- it is shown that for class A1 detectors, when determining the transition function, the sampling interval should not exceed $1.05 \mathrm{~s}$, and when determining the frequency characteristics, it should not exceed $0.27 \mathrm{~s}$.

The results obtained in the work can be used to conduct certification tests of heat fire detectors, as well as to monitor their technical condition during maintenance.

\section{Conclusions}

Methods have been developed for determining the dynamic characteristics of heat fire detectors, which are implemented in existing thermal chambers, as stipulated by the NFPA 72 and EN 54-5 standards. It justifies the choice of sampling intervals at which the error in determining the dynamic characteristics of heat fire detectors does not exceed the standard values. When determining the transition function of heat fire detectors with a time constant $\tau=20 \mathrm{~s}$, it is necessary to use the sampling interval $\tau_{0} \leq 1.05 \mathrm{~s}$. When determining the amplitude-frequency characteristic of such detectors, the sampling interval should be $\tau_{0} \leq 0.27 \mathrm{~s}$, and when determining the phase-frequency characteristic it is sufficient to use the sampling interval $\tau 0 \leq 2.00 \mathrm{~s}$. For the implementation of such methods for determining the HFD dynamic characteristics, a necessary and sufficient condition is the formation of a thermal test effect in the form of a linearly increasing function of time.

\section{References}

[1] Jemeljanov, V., Sulojeva, J., Bartusauskis, J. (2012). Analysis of the Inertial Parameters of Fire Detectors. Safety of Technogenic Environment, 3, 26-32.

[2] Wang, J., Xu, T. (2013). A New Calculation Model of Detection Time for Heat Detector in Long and Narrow Space. Procedia Engineering, 52, 355-362. doi: https://doi.org/10.1016/j.proeng.2013.02.153 
[3] Yamauchi, Y., Mammoto, A., Dohi, M., Ebata, H., Morita, M. (2005). A Calculation Method for Predicting Heat and Smoke Detector's Response. Fire Science and Technology, 24 (4), 179-210. doi: https:// doi.org/10.3210/fst.24.179

[4] Nam, S. (2006). Predicting response times of fixed-temperature, rate-of-rise, and rate-compensated heat detectors by utilizing thermal response time index. Fire Safety Journal, 41 (8), 616-627. doi: https:// doi.org/10.1016/j.firesaf.2006.06.004

[5] Qiang, L. (2011). Estimation of Fire Detection Time. Procedia Engineering, 11, 233-241. doi: https:// doi.org/10.1016/j.proeng.2011.04.652

[6] Gottuk, D. T., Pomeroy, A. T. (2011). Heat Detector RTI - New Developments, Fire Suppression and Detection Research and Applications - A Technical Working Conference, Orlando, Florida.

[7] Pomeroy, A. T. (2010).Analysis of the effects of temperature and velocity on the response time index of heat detectors. Available at: https://drum.lib.umd.edu/bitstream/handle/1903/10492/Pomeroy umd_0117N_11368.pdf?sequence=1

[8] Park, H.-W., Cho, J.-H., Mun, S.-Y., Park, C.-H., Hwang, C.-H., Kim, S.-C., Nam, D.-G. (2014). Measurement of the Device Properties of Fixed Temperature Heat Detectors for the Fire Modeling. Fire Science and Engineering, 28 (1), 37-43. doi: https://doi.org/10.7731/kifse.2014.28.1.037

[9] Abramov, Yu. O., Kalchenko, Ya. Yu. (2018). Pat. No. 125948 UA. Sposib vyprobuvan teplovykh pozhezhnykh spovishchuvachiv. MPK G08B 17/06. No. u201800548; declareted: 19.01.2018; published: 25.05.2018, Bul. No. 10.

[10] Kalchenko, Ya. Yu., Abramov, Yu. O., Sobyna, V. O. (2016). Pat. No. 111447 UA. Sposib vyznachennia dynamichnykh kharakterystyk teplovykh pozhezhnykh spovishchuvachiv. MPK G08B 29/00. No. u201604945; declareted: 04.05.2016; published: 10.11.2016, Bul. No. 21.

\title{
DEVELOPMENT OF A METHOD FOR DETERMINING THE OPTIMAL CONTROL TRAJECTORY FOR THE PERIODIC PROCESSES
}

\author{
Igor Lutsenko \\ Department of Automation and Information Systems \\ Kremenchuk Mykhailo Ostrohradskyi National University \\ 20 Pershotravneva str., Kremenchuk, Ukraine, 39600 \\ morev.igor11@gmail.com \\ Anatolii Oksanych \\ Department of Automation and Information Systems \\ Kremenchuk Mykhailo Ostrohradskyi National University \\ 20 Pershotravneva str., Kremenchuk, Ukraine, 39600 \\ oksirena2017@gmail.com \\ Olena Fomovskaya \\ Department of Electronic Devices \\ Kremenchuk Mykhailo Ostrohradskyi National University \\ 20 Pershotravneva str., Kremenchuk, Ukraine, 39600 \\ 2fill.fo@gmail.com \\ Iryna Oksanych \\ Department of Automation and Information Systems \\ Kremenchuk Mykhailo Ostrohradskyi National University \\ 20 Pershotravneva str., Kremenchuk, Ukraine, 39600 \\ oksirena2017@gmail.com
}

